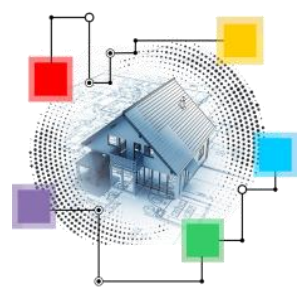

\author{
XIII SIMPÓSIO NACIONAL DE SISTEMAS PREDIAIS \\ DESEMPENHO E INOVAÇÃO \\ DE SISTEMAS PREDIAIS HIDRÁULICOS \\ SÃO PAULO - 04 DE OUTURO DE 2019
}

\title{
Avaliação da utilização de hidrômetros com aquisição de dados por telemetria para monitoramento do consumo de água de uma agência bancária em Joinville/SC
}

\section{Evaluation of the use of telemetry data acquisition meters to monitor water consumption at a bank branch in Joinville/SC}

\author{
LIMA, Marcio Ferreira de1; FREITAS, Lucas Lepinski Golin²; HENNING, Elisa3; \\ KALBUSCH, Andreza 4 \\ ${ }^{1}$ Gerência de Filial de Logística da Caixa Econômica Federal, marcio.f.lima@ caixa.gov.br. \\ 2 Dep.. de Eng. Civil - Universidade do Estado de Santa Catarina - UDESC, lgolin4@gmail.com. \\ ${ }^{3}$ Dep. de Matemática - Universidade do Estado de Santa Catarina - UDESC, elisa.henning@ @udesc.br. \\ ${ }^{4}$ Dep. de Eng. Civil - Universidade do Estado de Santa Catarina - UDESC, \\ andreza.kalbusch@udesc.br.
}

\section{RESUMO}

O objetivo deste artigo é avaliar a utilização de um hidrômetro com aquisição de dados por telemetria para monitoramento do consumo de água de uma agência bancária em Joinville/SC, para detecção de excessos de consumo e vazamentos. A avaliação do medidor ocorreu durante 6 meses, de novembro de 2018 até abril de 2019. O sensor acoplado ao hidrômetro registra uma leitura a cada 15 minutos, o que resulta 2.880 medições automáticas em 30 dias. Os dados são armazenados no registrador e sincronizados com servidor em nuvem, possibilitando acesso a distância. A análise estatística dos dados foi realizada com auxílio da linguagem de programação R. Foram desenvolvidos scripts para importação dos dados, formatação e análise. $\mathrm{Na}$ etapa de análise foram utilizados gráficos de controle estatístico de processos. A mediana de consumo diário foi de $5,925 \mathrm{~m}^{3}$, desvio padrão de $3,25 \mathrm{~m}^{3}$ e consumo máximo de $25,68 \mathrm{~m}^{3}$. Conclui-se que a gestão inteligente da água com uso de medidores de vazão associados a ferramentas de tecnologia da informação é uma inovação que auxilia na identificação quase que imediata de excessos de consumo. Leituras automáticas possibilitaram a elaboração de gráficos de controle em curtos períodos, possibilitando assim, detecção de anomalias de consumo e atuação mais rápida para redução do desperdício de água do imóvel.

Palavras-chave: Controle estatístico de processos, Consumo de água, Edifícios públicos, Linguagem R, Sistemas prediais hidrossanitários.

\begin{abstract}
The aim of this paper is to evaluate the use of a telemetry data acquisition hydrometer to monitor the water consumption of a bank branch in Joinville /SC, in order to detect excessive consumption and leakages. The measurement system trial lasted for 6 months, from November 2018 to April 2019. The sensor connected to the hydrometer registers readings every 15 minutes, resulting in a total of 2.880 automatic readings for every 30 days. The data is stored in a meter and synchronized in a cloud server, allowing easy access to the data anywhere. The statistical data analysis was executed using $R$ software programming language. Programming scripts were developed for importing, formatting and analyzing the data. Statistical process control charts were used in the analysis section. The median daily consumption was $5.925 \mathrm{~m}^{3}$, standard deviation of $3.25 \mathrm{~m}^{3}$ and maximum consumption of $25.68 \mathrm{~m}^{3}$. The conclusion is that the water management system with a flow rate measurement system, combined with information technology tools proved to be an innovation that helps to identify overconsumption almost instantly. Automatic readings made it possible to elaborate control charts in short periods, thus allowing the detection of consumption anomalies and faster action to reduce the water misuse.
\end{abstract}

Keywords: Statistical Process Control, Water Consumption, Public buildings, R language, Plumbing systems. 


\section{INTRODUÇÃO}

O $5^{\circ}$ relatório do Painel Intergovernamental sobre mudanças climáticas (Intergovernmental Panel on Climate Change - IPCC) prevê que as alterações climáticas no decorrer do século 21 reduzam significativamente os recursos hídricos superficiais e subterrâneos nas regiões subtropicais mais secas, intensificando a competição por água entre os setores (IPCC, 2014).

Muitos desafios complexos são enfrentados pela indústria da água hoje, sendo os vazamentos uma das maiores preocupações (WELSH, 2018). Água inteligente (em inglês, smart water) é a tendência de desenvolvimento da informação sobre a água (YUANYUAN et al., 2017). Conceitos sobre a Internet das Coisas, em inglês, Internetof-Things (IoT), foram aplicados em um estudo de caso de vazamentos de água por Maamar et al. (2019). A proposta de um sistema inteligente de gerenciamento de água para cidades inteligentes na Índia foi concebida por Mohammed Shahanas e Bagavathi Sivakumar (2016). A detecção remota de vazamentos de água em 47 residências do distrito de Point Vernon da cidade de Hervey Bay, Austrália, identificou vazamentos em bacias sanitárias com valor de 16,7 litros/hora (BRITTON et al., 2008).

Medidores de água inteligentes estão sendo cada vez mais instalados por empresas francesas de serviços de água e disponibilizados gratuitamente aos usuários. No entanto, segundo Montginoul e Vestier (2018), as famílias não estão aproveitando essa tecnologia, apesar dos benefícios que teoricamente oferece. Os autores concluem que há necessidade de reforçar a conscientização dos usuários sobre a importância da conservação da água.

O objetivo deste artigo é avaliar a utilização de um medidor inteligente de consumo de água em uma agência bancária em Joinville/SC, para detecção de excessos de consumo e vazamentos.

\section{METODOLOGIA}

A avaliação do medidor ocorreu durante 6 meses, de novembro de 2018 até abril de 2019 . O sistema é composto por um hidrômetro multijato (instalado após o medidor da concessionária local, sem interferência na medição existente), um sensor de leitura por saída de pulsos, um registrador de dados (data logger) e transmissão via rede de telefonia móvel GPRS para um servidor com acesso via navegador de internet.

O imóvel, objeto do estudo, é uma agência bancária localizada no centro da cidade de Joinville, em Santa Cantarina. Trata-se de um edifício com 4 pavimentos e área interna de $2.032,23 \mathrm{~m}^{2}$. O horário de funcionamento da agência é das $8 \mathrm{~h}$ às $18 \mathrm{~h}$ de segunda a sexta-feira e o horário de abertura ao público é das $11 \mathrm{~h}$ às $16 \mathrm{~h}$. A sala de autoatendimento funciona das $8 \mathrm{~h}$ às $20 \mathrm{~h}$, inclusive nos fins de semana e feriados.

O abastecimento de água potável é fornecido pela concessionária municipal. As instalações hidráulicas do edifício são compostas por um reservatório inferior de 30.000 litros e um reservatório superior de 20.000 litros. O edifício possui 23 bacias sanitárias com acionamento por válvula de descarga, 6 mictórios e 24 lavatórios com torneiras de fechamento automático por pressão, 3 tanques para limpeza geral com torneira convencional e uma torneira externa com chave de uso restrito usada para limpeza de calçada. Outro ponto relevante de consumo de água é o sistema de ar condicionado central, tipo chiller, com torre de resfriamento de água.

O sensor acoplado ao hidrômetro registra uma leitura a cada 15 minutos, o que resulta 2.880 medições automáticas em 30 dias. Os dados são armazenados no registrador e sincronizados com servidor em nuvem, possibilitando acesso à distância. O sistema está 
configurado para uma sincronização diária à 00h00.

A gestão de infraestrutura da agência bancária, objeto do estudo, e das demais agências localizadas no estado de Santa Catariana é realizada de maneira centralizada na capital do estado. Os serviços prestados pelo setor de engenharia do banco incluem a gestão dos contratos de fornecimento de água e energia elétrica, junto às concessionárias locais e contratos com empresas especializadas em serviços de limpeza e manutenção predial. As rotinas de manutenção predial incluem ações preventivas com vistorias mensais para localização de vazamentos e ações corretivas para conserto dos aparelhos hidráulicos que apresentarem vazamentos identificados pelos usuários do imóvel.

Os dados, com as leituras de consumo de água a cada 15 minutos, foram exportados em arquivos de texto com valores separados por vírgulas, extensão .CSV (comma to separate values, em inglês). A análise estatística dos dados foi realizada com auxílio da linguagem de programação R (R CORE TEAM, 2019). Foram desenvolvidos scripts para importação dos dados, formatação e análise. Na etapa de análise foram utilizados gráficos de controle estatístico.

O princípio básico de um processo sob controle estatístico é que este sempre está sujeito a variações provenientes de causas aleatórias. Dentre todas as ferramentas para avaliar se um processo está sob controle o gráfico de controle estatístico é a ferramenta bastante poderosa. Um gráfico de controle genérico possui uma Linha Central horizontal (LC) representando o valor médio dos dados coletados correspondentes a um estágio do processo que está sob controle. Existem ainda duas outras linhas horizontais, denominadas Limite de Controle Superior (LCS) e Limite de Controle Inferior (LCI). Estes limites de controle determinam se o processo está sob controle, considerando uma situação em que quase todos os pontos amostrais estejam contidos entre os limites LCS e LCI. Caso um ponto ultrapasse algum dos limites, considera-se que o processo não está sob controle estatístico e sugere-se investigar as causas (MONTGOMERY; RUNGER, 2013).

Dentre os diversos modelos de gráficos de controle existentes, destaca-se o gráfico de controle de Shewhart, popular devido a sua fácil interpretação e eficácia para identificar mudanças bruscas e repentinas em um processo. Assumindo que as observações originais sejam $\mathrm{x}_{1}, \mathrm{x}_{2}, \ldots . \mathrm{x}_{\mathrm{n}}$, independentes e com distribuição normal e assumindo que $\mathrm{x}_{\mathrm{m}}$ seja a média amostral das observações originais, o cálculo das linhas LCS, LC e LCI para os gráficos de Shewhart são expressos pelas equações 1 a 7 .

$$
\begin{aligned}
& L C S=x_{m}+E_{2} * \overline{M R} \\
& L C=x_{m} ; \\
& L C I=x_{m}-E_{2} * \overline{M R} \\
& x_{m}=\frac{\sum_{i=1}^{m} x_{i}}{m}
\end{aligned}
$$

onde $x_{m}$ é a média das medidas individuais $\left(x_{i}\right)$. Em sequência define-se:

$\overline{M R}=\left(\frac{1}{n}\right) * \sum_{i=1}^{n} \overline{M R_{1}}$

sendo $\overline{M R}$ a média aritmética dos valores de

$\overline{M R_{1}}=\left|x_{i}-x_{i-1}\right|$ para $\mathrm{i}=1,2 \ldots \mathrm{n}$; 
onde $\overline{M R_{1}}$ é a diferença entre uma medida $x_{i}$ e sua respectiva medida anterior $x_{i-1}$

e finalmente $E_{2}=\frac{3}{d_{2}}$

é uma constante tabelada, que depende do número de amostras (m). Para gráficos de controle de medidas individuais utilizados neste trabalho, adota-se um tamanho de amostra $\mathrm{n}=1$. Desta forma, o valor encontrado de $E_{2}=2,66$ utilizado nesse artigo é obtido divindo-se 3 por uma constante amostra de tamanho específico $d_{2}$ com $n=2$ (MONTGOMERY; RUNGER, 2013).

\section{RESULTADOS E DISCUSSÃO}

Foram realizadas análises diárias do consumo do dia anterior com objetivo de identificar variações no perfil de consumo, sejam excessos de consumo justificáveis por rotinas de limpeza e manutenção ou vazamentos nos sistemas prediais hidráulicos. Os excessos identificados foram comunicados à equipe de manutenção predial para realização de vistorias, identificação de vazamentos e correção.

A Tabela 1 apresenta as medidas descritivas dos dados coletados diariamente, por hora e a cada 15 minutos, no período de novembro de 2018 até abril de 2019.

TABELA 1 - Medidas descritivas

\begin{tabular}{c|c|c|c|c|c}
\hline Consumo $\left(\mathbf{m}^{3}\right)$ & Mínimo & Máximo & Mediana & Média & Desvio Padrão \\
\hline Diário & 0 & 25,68 & 5,925 & 5,264 & 3,25 \\
\hline Horário & 0 & 1,73 & 0,09 & 0,219 & 0,264 \\
\hline 15 Minutos & 0 & 0,44 & 0,02 & 0,055 & 0,067 \\
\hline
\end{tabular}

No primeiro mês de uso do sistema, entre os dias 30 de outubro e 30 de novembro de 2018, foi registrado o consumo mensal de $163 \mathrm{~m}^{3}$ e consumo médio diário de 5,06 $\mathrm{m}^{3}$ no período, conforme detalhado na Figura 1. Nesse mês ocorreram dois feriados, em 02/11/18 e em 15/11/18.

No feriado do dia 02/11/18 ocorreu um pico de consumo de 11,47 $\mathrm{m}^{3}$. Não foi identificado o motivo deste consumo, pois o sistema de monitoramento estava na fase inicial de operação, sem dados suficientes para análise. No dia seguinte (sábado), o consumo foi de $3 \mathrm{~m}^{3}$ e no domingo, de $0,76 \mathrm{~m}^{3}$. Este padrão de consumo se repetiu no fim de semana de 09 e 10/11/18 com consumo de 3,87 e $0,76 \mathrm{~m}^{3}$, respectivamente. Os limites de controle estão representados na cor vermelha e verifica-se que o processo se encontra sob controle estatístico.

Devido à natureza estatística dos gráficos de controle as linhas LCS e LCI estão sempre presentes. Todavia, neste trabalho optou-se por fixar a linha LCI em 0, pois neste estudo, nos gráficos elaborados a linha LCI se encontrava com um valor negativo que não apresenta nenhum significado no processo analisado (representaria consumo negativo de água). No entanto em outros estudos envolvendo consumo de água o LCI pode ser importante para sinalizar redução de consumo devido à adoção de equipamentos economizadores de água, como no estudo de FREITAS et al., (2019), ou até mesmo por conta de períodos de escassez ou restrição de abastecimento. 


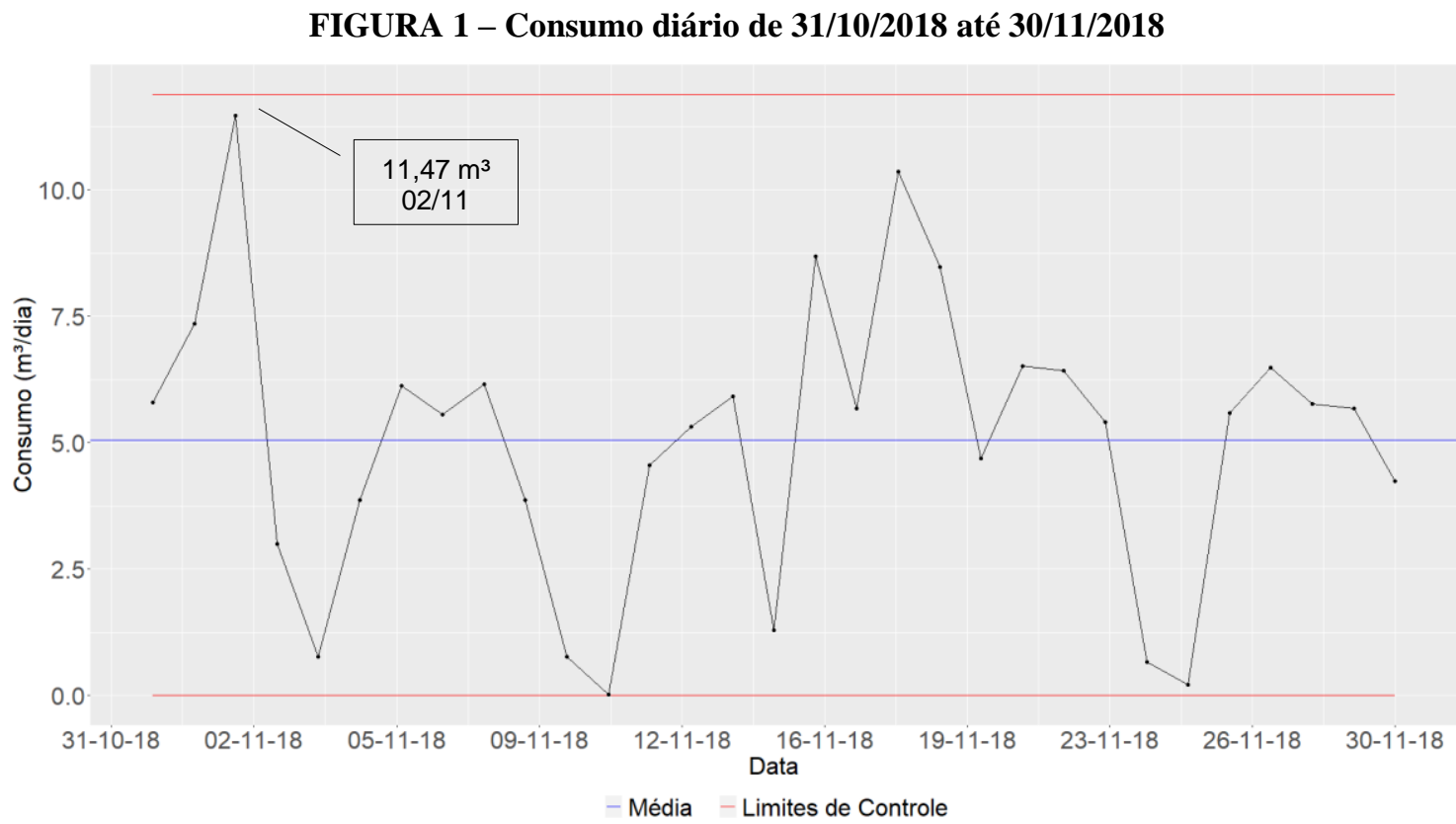

Nos fins de semana e feriados não há funcionários na agência, apenas a sala de autoatendimento fica aberta ao público. A rotina diária de limpeza do piso é mantida nestes dias, podendo ser realizada mais de uma vez por dia, dependendo da necessidade, principalmente em dias de chuva. Outro consumo identificado são obras de manutenção e reforma que ocorrem fora do horário de atendimento ao público.

Estas rotinas de limpeza e manutenção justificam o consumo de água nestes períodos. Embora tenham sido registrados consumos elevados de até $10,36 \mathrm{~m}^{3}$ no domingo, 18/11/18, que sugerem outros usos mais intensos ou até mesmo vazamentos.

O consumo permaneceu constante durante o mês de dezembro de 2018 e janeiro de 2019 , exceto por um consumo excessivo de $25,68 \mathrm{~m}^{3}$ no dia 30 de janeiro de 2019 , conforme demonstrado na Figura 2. Este ponto aparece além do LCS.

FIGURA 2 - Consumo diário de 30/10/2018 até 08/02/2019

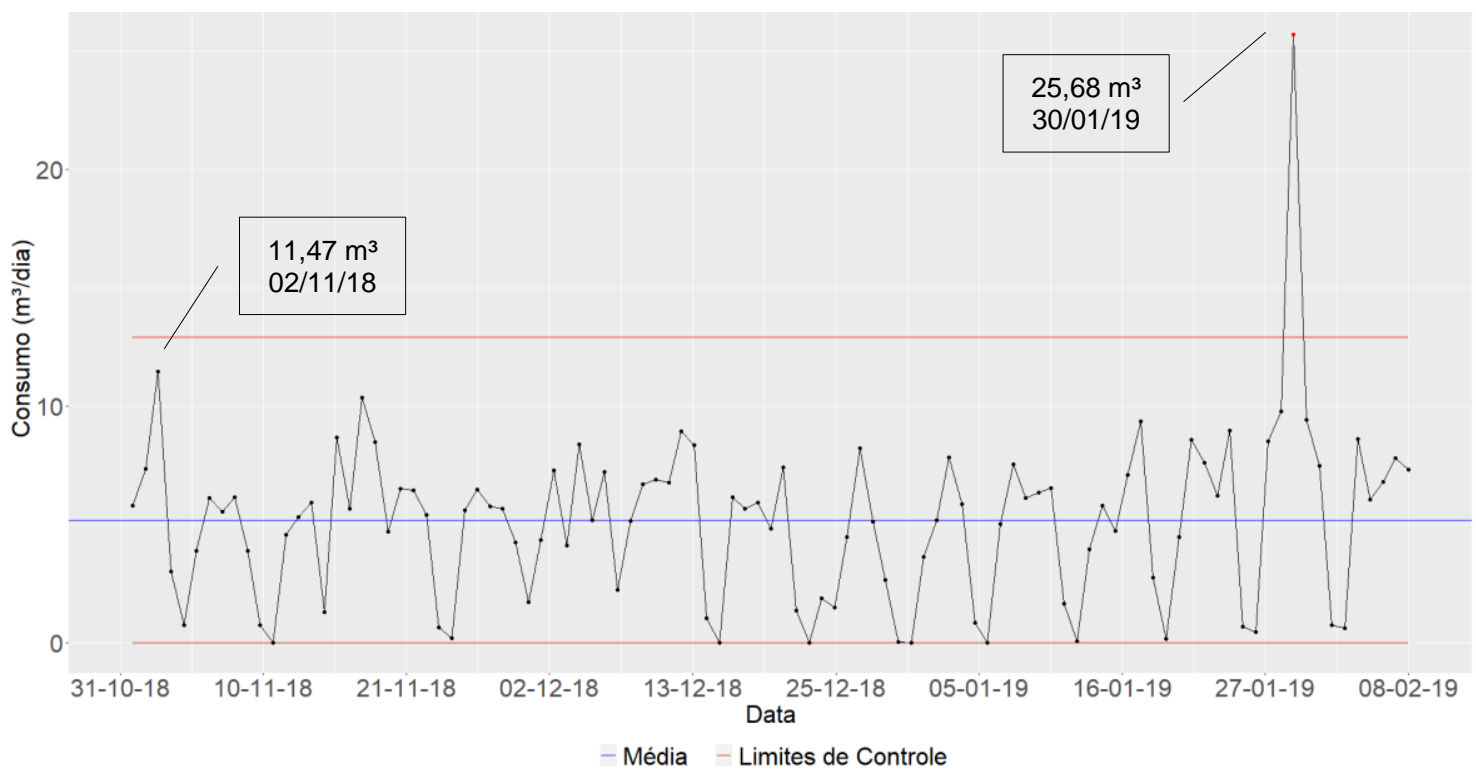


Observando o consumo horário do dia 27/01/19 a 03/02/19 (Figura 3), no domingo, 27/01/19, não houve consumo. Na segunda-feira (28/01/19) o consumo foi normal, com picos de $0,8 \mathrm{~m}^{3}$ por hora, reduzindo a partir das 18 horas. Na terça-feira (29/01/19) o consumo não reduziu como esperado, tendo aumentado até o pico de $1,73 \mathrm{~m}^{3} / \mathrm{h}$ às $04 \mathrm{~h} 00$ do dia 30/01/19 e então começou a reduzir. O consumo diário voltou ao perfil normal na quinta e sexta-feira (31/01/19 e 01/02/19), com redução do consumo após às $18 \mathrm{~h} 00$. No sábado (dia 02/02/19) o consumo foi próximo de zero.

Não foi registrado, pelos funcionários da agência, nenhum chamado para acionamento da empresa de manutenção predial para conserto de vazamentos no dia 30/01/19. A causa mais provável, segundo o gerente da agência, é o uso indevido de uma torneira externa, localizada no jardim, por moradores de rua durante a madrugada. Foi providenciada a retirada da mangueira e a instalação de uma grade de proteção com cadeado para impedir o uso indevido da torneira.

FIGURA 3 - Consumo horário de 27/01/2019 até 03/02/2019

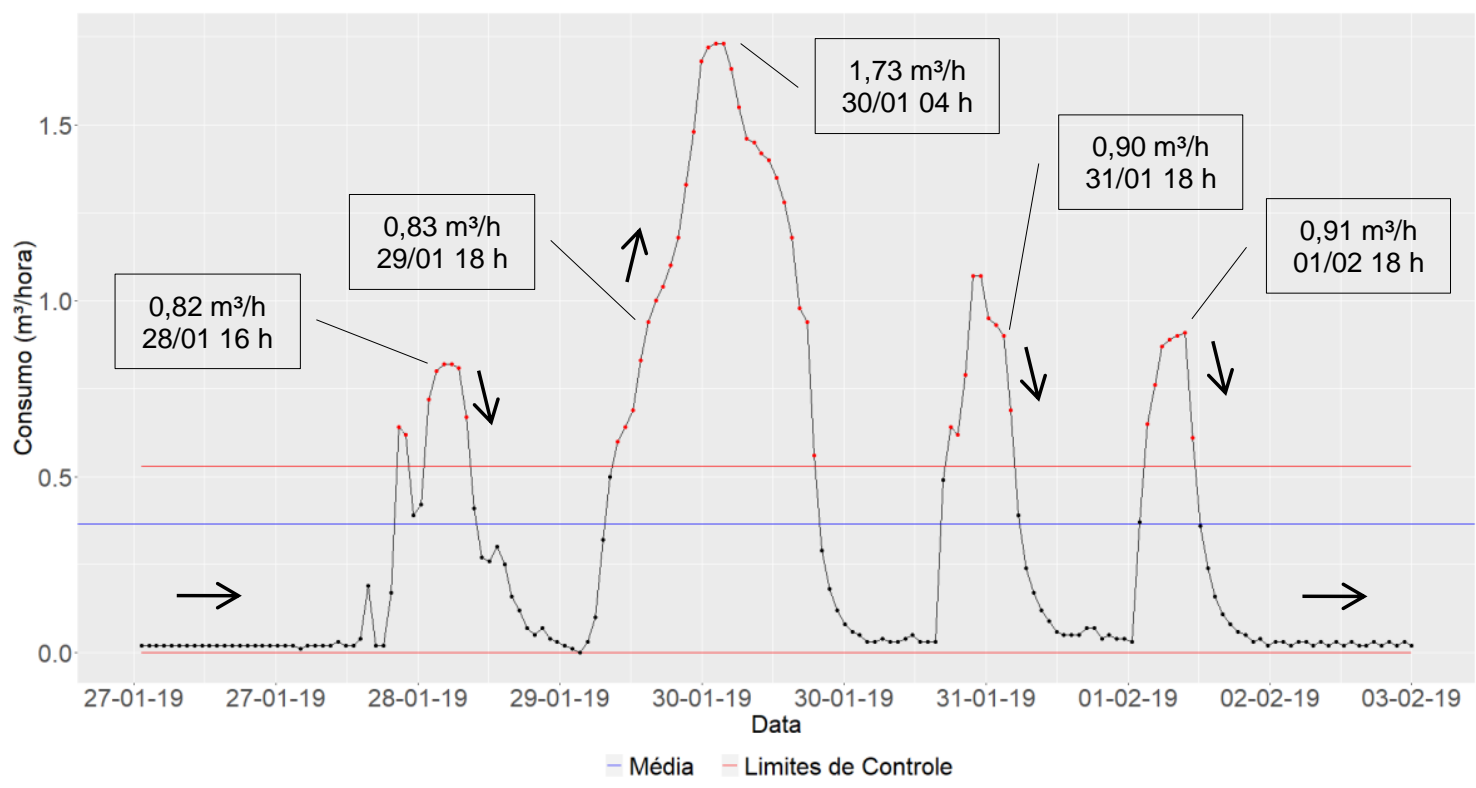

Na Figura 4 está representado o consumo diário em todo o período do estudo, entre 31/10/18 e 30/04/2019, destaca-se acima do Limite de Controle Superior (LCS) o pico de consumo de $25,68 \mathrm{~m}^{3}$ no dia 30/01/2019. 
FIGURA 4 - Consumo diário de 31/10/2018 até 30/04/2019

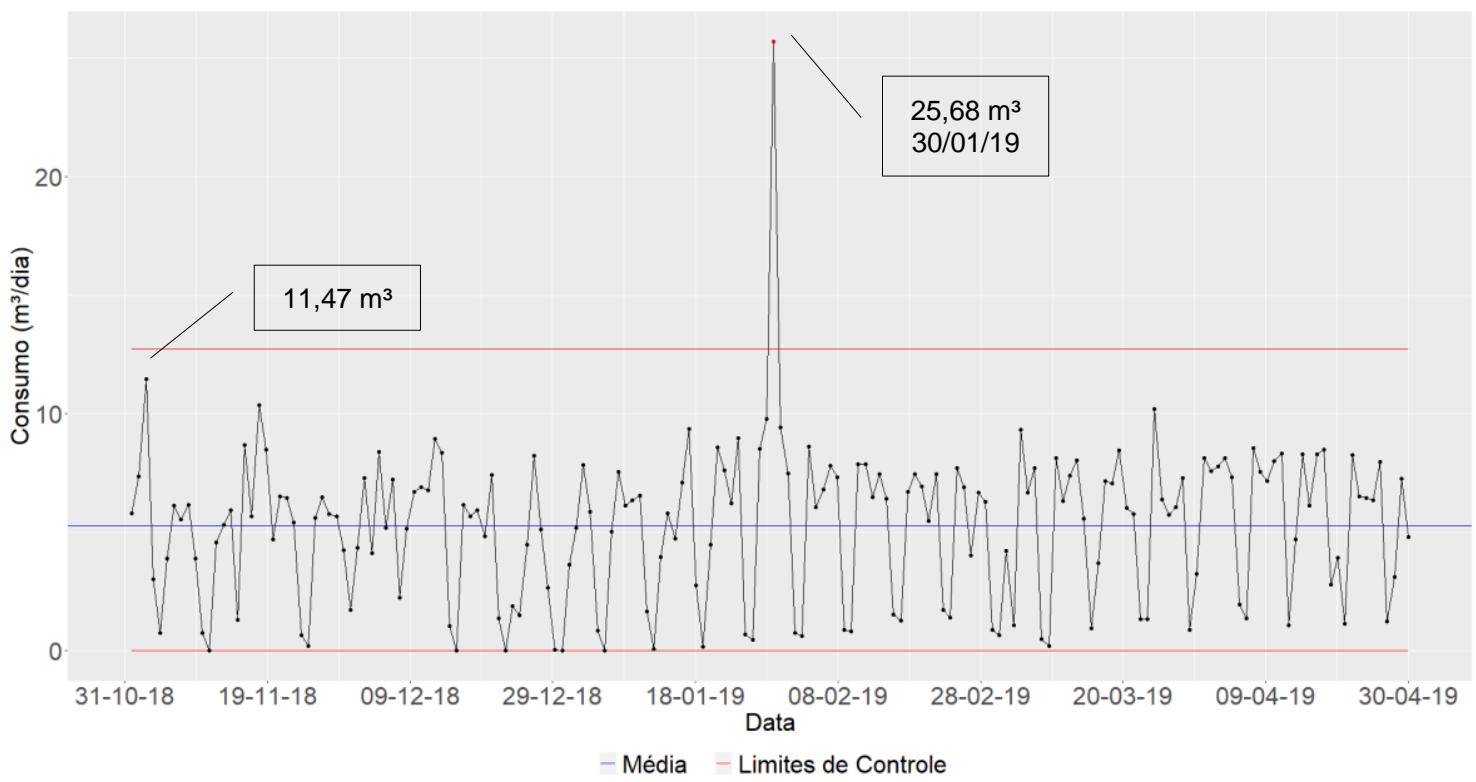

\section{CONCLUSÕES}

A gestão inteligente da água com uso de medidores de vazão associados a ferramentas de tecnologia da informação, com recursos de leitura do consumo em tempo real, transmissão dos dados via rede de telefonia móvel e consulta de informações disponíveis na internet para tomada de decisões é uma inovação que auxilia na identificação quase que imediata de excessos de consumo. Desta forma, pode ser quebrado o paradigma atual, de aguardar a fatura mensal da concessionária de fornecimento de água para analisar o volume consumido.

A atuação diária na análise do consumo possibilitou a identificação de excessos de consumo em apenas um dia, logo após o início do problema. Os gráficos de controle auxiliaram na identificação de excessos de consumo, por meio da LCS. Leituras automáticas possibilitaram a elaboração de gráficos de controle em curtos períodos, possibilitando assim, detecção de anomalias de consumo e atuação mais rápida para redução do desperdício de água do imóvel. Nas vistorias das equipes de manutenção não foram localizados vazamentos.

Sugere-se, para trabalhos futuros, a elaboração de scripts com alertas automáticos (por exemplo, via e-mail) para notificar imediatamente as equipes de manutenção predial e possibilitar a atuação quando da ocorrência de excessos de consumo ou vazamentos.

\section{REFERÊNCIAS}

BRITTON, T. C.; COLE, G.; STEWART, R. A.; WISKAR, D. Remote Diagnosis of Leakage in Residential Households. Water, n. September, p. 56-60, 2008.

FREITAS, L. L. G.; HENNING, E.; KALBUSCH, A.; KONRATH, A. C.; WALTER, O. M. F. C. Analysis of water consumption in toilets employing Shewhart, EWMA, and ShewhartEWMA combined control charts. Journal of Cleaner Production, v. 233, p. 1146-1157, 2019.

IPCC. Summary for policymakers. In: FIELD, C.B., V.R. BARROS, D.J. DOKKEN, K. J. M.; M.D. MASTRANDREA, T.E. BILIR, M. CHATTERJEE, K.L. EBI, Y.O. ESTRADA, R.C. 
GENOVA, B. GIRMA, E.S. KISSEL, A. N.; LEVY, S. MACCRACKEN, P. R. M. E L. L. W. (Eds.). . Climate Change 2014: Impacts, Adaption, and Vulnerability.Part A: Global and Sectoral Aspects. Cambridge, UK and New York, USA: Cambridge University Press, 2014. p. $1-32$.

MAAMAR, Z.; BAKER, T.; FACI, N.; AL-KHAFAJIY, M.; UGLJANIN, E.; ATIF, Y.; SELLAMI, M. Weaving cognition into the internet-of-things: Application to water leaks. Cognitive Systems Research, v. 56, p. 233-245, ago. 2019.

MOHAMMED SHAHANAS, K.; BAGAVATHI SIVAKUMAR, P. Framework for a Smart Water Management System in the Context of Smart City Initiatives in India. Procedia Computer Science, v. 92, p. 142-147, 2016.

MONTGINOUL, M.; VESTIER, A. Smart metering: A water-saving solution? Consider communication strategies and user perceptions first. Evidence from a French case study. Environmental Modelling \& Software, v. 104, p. 188-198, jun. 2018.

MONTGOMERY, D. C.; RUNGER, G. C. Estatística aplicada e probabilidade para engenheiros. Rio de Janeiro: LTC, 2013.

R CORE TEAM. R: A Language and Environment for Statistical Computing. R Foundation for Statistical Computing, Vienna, Austria, Disponível em: 〈https://www.r-project.org/>. Acesso em: 14 maio. 2019

WELSH, A. Smarter water infrastructure. World Pumps, v. 2019, n. 1, p. 22-23, 1 jan. 2018.

YUANYUAN, W.; PING, L.; WENZE, S.; XINCHUN, Y. A New Framework on Regional Smart Water. Procedia Computer Science, v. 107, n. Icict, p. 122-128, 2017. 